\title{
INSTITUIÇÃo PSIQUiÁTRICA DE LONGA PERMANÊNCIA: PERFIL DE PACIENTES E INDICADORES DE RECURSOS HUMANOS
}

\author{
Maria Odete Pereira ${ }^{1}$ \\ Marli de Carvalho Jericó ${ }^{2}$ \\ Marcia Galan Perroca ${ }^{3}$ \\ Helena Ayako Mukai ${ }^{4}$
}

Este estudo descritivo teve como objetivo investigar o perfil sociodemográfico e epidemiológico de pacientes e indicadores de recursos humanos em um hospital neuropsiquiátrico, localizado na Região Sudeste do Brasil. Os dados relativos aos indicadores de recursos humanos (2006-2010) e caracterização sociodemográfica e epidemiológica dos pacientes (2010) foram extraídos da base de dados do sistema de gestão hospitalar e planilhas de escala de pessoal. Participaram do estudo 105 pacientes com diagnóstico de transtorno mental. A maioria era do sexo masculino $(60 ; 57,1 \%)$, idade média 52,5 (11,4) anos, tempo de internação até 15 anos $84(80 \%)$ e diagnóstico principal de esquizofrenia $50(47,7 \%)$ e retardo mental 41 (39\%). A relação enfermagem/leito variou de 0,52 a 1,15 e a de enfermeiro/leito de 0,05 a 0,11 . Almeja-se que os resultados desta investigação possam contribuir para a elaboração de políticas de saúde mental e instrumentalizar o gestor na tomada de decisão e estabelecimento de estratégias de ação.

Descritores: Pessoas Mentalmente Doentes; Enfermagem Psiquiátrica; Indicadores de Qualidade em Assistência à Saúde.

\footnotetext{
${ }^{1}$ PhD, Professor, Universidade Federal de São Paulo, São Paulo, SP, Brasil.

${ }^{2} \mathrm{PhD}$, Professor, Faculdade de Medicina de São José do Rio Preto, São José do Rio Preto, SP, Brasil.

${ }^{3} \mathrm{PhD}$, Professor Adjunto, Faculdade de Medicina de São José do Rio Preto, São José do Rio Preto, SP, Brasil.

${ }^{4}$ MSc, Professor, Centro Estadual de Educação Tecnológica Paula Souza, Lins, SP, Brasil.
} 


\section{Long Term Psychiatric Institution: Profile of Patients and human RESOURCE INDICATORS}

This descriptive study aimed to investigate the social-demographic and epidemiological profile of patients and human resource indicators in a neuropsychiatric hospital located in southeastern Brazil. The data related to the human resources (2006-2010) and the socialdemographic and epidemiological characterization of the patients (2010) were extracted from the hospital management system database and staff scheduling worksheets. The study included 105 patients with a mental disorder diagnosis. Most were male 60 (57.1\%), mean age 52.5 (11.4) years, duration of hospitalization up to 15 years $84(80 \%)$, and primary diagnosis of schizophrenia $50(47.7 \%)$, and mental retardation $41(39 \%)$. The nursing staff/bed ratio ranged from 0.52 to 1.15 and the nurse/bed from 0.05 to 0.11 . The aim of this research is to contribute to the development of mental health policies and equip the administrator for the strategic decision making processes.

Descriptors: Mentally Ill Persons; Psychiatric Nursing; Quality Indicators, Health Care.

\section{Institución Psiquiátrica de Larga Permanencia: Perfil de Pacientes e INDICADORES DE RECURSOS HUMANOS}

Este estudio descriptivo tuvo como objetivo investigar el perfil sociodemográfico y epidemiológico de pacientes e indicadores de recursos humanos en un hospital neuro psiquiátrico localizado en la región sudeste de Brasil. Los datos relativos a los indicadores de recursos humanos (2006-2010) y caracterización socio demográfica y epidemiológica de los pacientes (2010) fueron extraídos de la base de datos del sistema de gestión hospitalaria y planillas de escala de personal. Participaron del estudio 105 pacientes con diagnóstico de trastorno mental. La mayoría era del sexo masculino 60 $(57,1 \%)$, edad media 52,5 $(11,4)$ años, tiempo desde internación hasta 15 años $84(80 \%)$ y diagnóstico principal de esquizofrenia 50(47,7\%) y retardo mental 41(39\%). La relación enfermería/camas varió de 0,52 a 1,15 y la enfermero/camas de 0,05 a 0,11. El objetivo es que los resultados de esa averiguación puedan aportar en la elaboración de políticas de salud mental e instrumentalizar el gestor en la tomada de decisión y establecimiento de estrategias de acción.

Descriptores: Enfermos Mentales; Enfermería Psiquiátrica; Indicadores de Calidad de la Atención de Salud.

\section{Introdução}

A assistência psiquiátrica, até a segunda metade do século XX, baseou-se principalmente na hospitalização e no asilamento do doente mental. Em 1987, iniciouse a Reforma Psiquiátrica no Brasil que objetivava a regulamentação dos direitos da pessoa com transtorno mental e a extinção progressiva dos manicômios. A redução planejada e programada de leitos deveria ser acompanhada pela expansão da rede comunitária, com a implantação 
de serviços substitutivos e credenciamento de leitos psiquiátricos em hospitais gerais, quando necessário ${ }^{(1)}$.

Dados do Ministério da Saúde ${ }^{(2)}$ revelam que, no ano 1996, existiam 72.514 leitos psiquiátricos, os quais foram reduzidos para 42.076 em 2005, porém, concentrados nos grandes centros, especialmente na Região Sudeste - 60\%.

A assistência hospitalar em psiquiatria tomou novos rumos a partir da Portaria 251/GM, 2002 $2^{(1)}$, que estabeleceu diretrizes e normas para melhorar a qualidade dos serviços hospitalares prestados, respeitando os princípios de universalidade e da equidade. Foi então criado o Programa Nacional de Avaliação do Sistema Hospitalar/Psiquiatria (PNASH) que definiu indicadores de qualidade para classificação hospitalar.

$\mathrm{O}$ indicador constitui-se em medida quantitativa utilizada como um guia para monitorar e avaliar a assistência $^{(3)}$. Sua utilização possibilita replanejar e reorganizar as atividades de um serviço, oferecendo subsídios para tomada de decisões ${ }^{(4)}$. O uso sistematizado de indicadores norteia a melhoria contínua da qualidade assistencial e aumento da produtividade, beneficiando pacientes, profissionais e administração ${ }^{(5)}$.

A desinstitucionalização da pessoa com transtorno mental e sua consequente reinserção social modificaram o perfil dessa população. Dessa forma, pesquisas que abordam essa temática contribuem para o planejamento e organização dos serviços constituintes da rede de assistência psiquiátrica e aprimoramento das políticas assistenciais $^{(6)}$.

Observa-se que, após a Reforma Psiquiátrica, alguns estudos têm investigado as características sociodemográficas e epidemiológicas de pacientes em diferentes cenários: instituição de longa permanência ${ }^{(7)}$, Centro de Atenção Psicossocial (Caps) ${ }^{(8)}$, Hospital-dia( ${ }^{(9)}$, instituição de curta permanência ${ }^{(6,10)}$ e instituições de curta e longa permanência ${ }^{(11-12)}$.

\section{Objetivos}

Investigar o perfil sociodemográfico e epidemiológico de pacientes e indicadores de recursos humanos em hospital neuropsiquiátrico.

\section{Método}

\section{Delineamento e sujeitos}

Trata-se de pesquisa descritiva com delineamento transversal. Foi desenvolvida em um hospital neuropsiquiátrico público de grande porte, localizado na Região Sudeste do Brasil. A instituição atende essencialmente pacientes do Sistema Único de Saúde (SUS), com área de abrangência do Departamento Regional de Saúde VI (DRS-VI) de Bauru. Atualmente, conta com 340 leitos de internação integrais, sendo 160 leitos destinados a pacientes psiquiátricos e 180 leitos para pacientes neurológicos.

O quadro de pessoal é composto por 696 funcionários, sendo 199 das áreas administrativas e 497 da área assistencial. A equipe de enfermagem encontrase constituída por 31 enfermeiros e 298 auxiliares de enfermagem. Dentre os demais profissionais atuantes, encontram-se 32 médicos (cinco psiquiatras e dois neurologistas), quatro fisioterapeutas, dois fonoaudiólogos, três cirurgiões dentistas, dez psicólogos, onze assistentes sociais, três farmacêuticos e onze terapeutas ocupacionais.

Foram objetos de investigação somente os pacientes com transtornos mentais internados nas Gerências de Atenção Integral à Saúde (Gais) IV e Núcleo de Moradias Protegidas (NMP), totalizando 105 pacientes distribuídos em três unidades de internação. As unidades F1 e F3 totalizam 70 leitos e destinam-se ao atendimento de moradores com transtornos mentais persistentes e severos, com história de vários anos de internação. A unidade C3 (40 leitos) faz parte da NMP e dispõe de oito apartamentos destinados a pacientes com menor grau de dependência.

No que se refere às variáveis a serem estudadas, elas se constituem em:

1-sociodemográficas - sexo, idade, escolaridade, estado civil, fonte de renda, procedência e tempo de internação;

2-epidemiológicas - diagnóstico principal, comorbidades, terapêutica medicamentosa de uso contínuo;

3-indicadores de recursos humanos - relação enfermagem/ leito, enfermeiro/leito e auxiliar de enfermagem/leito.

\section{Procedimentos para coleta de dados}

A coleta de dados somente foi conduzida após autorização formal do hospital e Comitê de Ética em Pesquisa da Faculdade de Medicina de São José do Rio Preto (Parecer n⿳319/2010). Os dados relativos aos indicadores de recursos humanos (2006-2010) e caracterização sociodemográfica e epidemiológica dos pacientes (2010) foram extraídos da base de dados do sistema de gestão hospitalar e planilhas de escala de pessoal, disponibilizadas pela diretoria de enfermagem.

Os diagnósticos foram expressos de acordo com a Classificação Estatística Internacional de Doenças e Problemas Relacionados à Saúde (CID-10). O custo dos medicamentos foi obtido da farmácia. Os indicadores de recursos humanos foram calculados, segundo recomendações do Compromisso com a Qualidade Hospitalar $(\mathrm{CQH})^{(13)}$.

\section{Apresentação e Tratamento dos Dados}

Os dados obtidos foram submetidos a tratamento estatístico pelo The $R$ Foundation for Statistical Computing, versão 2.13.0. A análise descritiva encontrase apresentada como frequência absoluta, média, desviopadrão e porcentagem.

\section{Resultados}

Houve predomínio do sexo masculino $(60 ; 57,1 \%)$, faixa etária entre 41 e 60 anos $(61 ; 58,1 \%)$, idade média de $52,5(11,4)$ anos, variação - 18 a 80 anos, analfabetos 60 $(57,1 \%)$, solteiros $94(89,5 \%)$, sem qualquer tipo de renda $71(67,7 \%)$, procedentes da região de Bauru $72(68,6 \%)$ e 
com tempo de internação até 15 anos 84 (80\%). Em relação às unidades de internação, houve maioria feminina $(18$; $69 \%)$ e faixa etária de $61-80$ anos $(3 ; 11,5 \%)$ na unidade
F3. O tempo de internação mais prolongado (26-29 anos) teve maior ocorrência na unidade C3 $(5 ; 13 \%)$ e o menos prolongado ( $\leq 5$ anos) na F3 $(9 ; 34,6 \%)$ (Tabela 1$)$.

Tabela 1 - Distribuição das variáveis sociodemográficas dos pacientes internados, segundo unidades de internação (N=105). Lins, SP, Brasil, 2010

\begin{tabular}{|c|c|c|c|c|c|c|c|c|}
\hline \multirow{3}{*}{ Variáveis } & \multirow{2}{*}{\multicolumn{2}{|c|}{$\begin{array}{c}F 1 \\
(n=40)\end{array}$}} & \multirow{2}{*}{\multicolumn{2}{|c|}{$\begin{array}{c}F 3 \\
(n=26)\end{array}$}} & \multirow{2}{*}{\multicolumn{2}{|c|}{$\begin{array}{c}\text { C3 } \\
(n=39)\end{array}$}} & \multirow{2}{*}{\multicolumn{2}{|c|}{$\begin{array}{c}\text { Total } \\
(\mathrm{N}=105)\end{array}$}} \\
\hline & & & & & & & & \\
\hline & $\mathbf{n}$ & $\%$ & $\mathbf{n}$ & $\%$ & $\mathbf{n}$ & $\%$ & $\mathbf{n}$ & $\%$ \\
\hline \multicolumn{9}{|l|}{ Gênero } \\
\hline Feminino & 11 & 27,5 & 18 & 69,0 & 16 & 41,0 & 45 & 42,9 \\
\hline Masculino & 29 & 72,5 & 8 & 31,0 & 23 & 59,0 & 60 & 57,1 \\
\hline \multicolumn{9}{|l|}{ Faixa etária (anos) } \\
\hline$\leq 20$ & - & - & 1 & 3,9 & - & - & 1 & 0,9 \\
\hline $21-40$ & 3 & 7,5 & 9 & 34,6 & 3 & 7,7 & 15 & 14,3 \\
\hline $41-60$ & 27 & 67,5 & 13 & 50,0 & 21 & 53,8 & 61 & 58,1 \\
\hline $61-80$ & 10 & 25,0 & 3 & 11,5 & 15 & 38,5 & 28 & 26,7 \\
\hline \multicolumn{9}{|l|}{ Escolaridade } \\
\hline Analfabeto & 23 & 57,5 & 15 & 57,7 & 22 & 56,4 & 60 & 57,1 \\
\hline $1^{\circ} \mathrm{grau}$ incompleto & 17 & 42,5 & 11 & 42,3 & 17 & 43,6 & 45 & 42,9 \\
\hline \multicolumn{9}{|l|}{ Estado civil } \\
\hline Casado/união consensual & 4 & 10,0 & 1 & 3,8 & 2 & 5,1 & 7 & 6,6 \\
\hline Solteiro & 35 & 87,5 & 23 & 88,5 & 36 & 92,3 & 94 & 89,5 \\
\hline Desquitado/viúvo & 1 & 2,5 & 1 & 3,8 & - & - & 1 & 2,0 \\
\hline Sem dados & - & - & 1 & 3,8 & 1 & 2,6 & 2 & 1,9 \\
\hline \multicolumn{9}{|l|}{ Fonte de renda } \\
\hline Aposentadoria & 2 & 5,0 & 1 & 3,8 & 1 & 2,6 & 4 & 3,8 \\
\hline $\mathrm{BPC}^{*}$ & 7 & 17,5 & 5 & 19,3 & 7 & 17,9 & 19 & 18,1 \\
\hline Pensão INSS & 6 & 15,0 & 1 & 3,8 & 4 & 10,3 & 11 & 10,5 \\
\hline Sem renda & 25 & 62,5 & 19 & 73,1 & 27 & 69,2 & 71 & 67,7 \\
\hline \multicolumn{9}{|l|}{ Procedência } \\
\hline DRS VI-Bauru & 29 & 72,5 & 17 & 65,5 & 26 & 66,7 & 72 & 68,6 \\
\hline Outras** & 11 & 27,5 & 9 & 34,5 & 13 & 33,3 & 33 & 31,4 \\
\hline \multicolumn{9}{|l|}{ T.de internação (anos) } \\
\hline$\leq 5$ & 7 & 17,5 & 9 & 34,6 & 9 & 23,0 & 25 & 23,8 \\
\hline $6-10$ & 16 & 40,0 & 6 & 23,0 & 10 & 25,6 & 32 & 30,5 \\
\hline $11-15$ & 10 & 25,0 & 7 & 27,0 & 10 & 25,6 & 27 & 25,7 \\
\hline $16-20$ & 3 & 7,5 & 2 & 7,7 & 4 & 10,2 & 9 & 8,6 \\
\hline $21-25$ & 3 & 7,5 & - & - & 1 & 2,6 & 4 & 3,8 \\
\hline $26-29$ & 1 & 2,5 & 2 & 7,7 & 5 & 13,0 & 8 & 7,6 \\
\hline
\end{tabular}

*Benefício de Prestação Continuada; **Grande São Paulo, Araçatuba, Campinas, Franca, Piracicaba, São José do Rio Preto e Sorocaba.

O diagnóstico principal (Tabela 2) predominante foi esquizofrenia $(50 ; 47,7 \%)$ mais frequente nas unidades F1 $(20 ; 50 \%)$ e C3 $(21 ; 53,8 \%)$ e o diagnóstico retardo mental $(41 ; 39 \%)$ na unidade F3 $(9 ; 34,6 \%)$. No que se refere às comorbidades, o tabagismo $(65 ; 33,5 \%)$, seguido da obesidade $(45 ; 23,2 \%)$, hipertensão arterial $(21 ; 10,8 \%)$ e diabetes mellitus $(18 ; 9,3 \%)$ foram predominantes.

Tabela 2 - Diagnóstico principal e comorbidades, segundo unidades de internação e gênero (N=105). Lins, SP, Brasil, 2010

\begin{tabular}{|c|c|c|c|c|c|c|c|c|}
\hline \multirow{3}{*}{ Variáveis } & \multicolumn{2}{|c|}{ F1 } & \multicolumn{2}{|c|}{ F3 } & \multicolumn{2}{|c|}{ C3 } & \multicolumn{2}{|c|}{ Total } \\
\hline & Masculino & Feminino & Masculino & Feminino & Masculino & Feminino & Masculino & Feminino \\
\hline & $n(\%)$ & n (\%) & $n(\%)$ & $n(\%)$ & n (\%) & $n(\%)$ & n (\%) & n (\%) \\
\hline \multicolumn{9}{|l|}{ Diagnóstico principal } \\
\hline Esquizofrenia & $15(37,5)$ & $5(12,5)$ & $2(7,9)$ & $7(26,9)$ & $10(25,6)$ & $11(28,2)$ & 50 & $(47,7)$ \\
\hline Ret.mental & $9(22,5)$ & $6(15,0)$ & $4(15,4)$ & $9(34,6)$ & $9(23,0)$ & $4(10,2)$ & 41 & $(39,0)$ \\
\hline
\end{tabular}


Table 2 - (continuation)

\begin{tabular}{|c|c|c|c|c|c|c|c|c|}
\hline \multirow{3}{*}{ Variáveis } & \multicolumn{2}{|c|}{ F1 } & \multicolumn{2}{|c|}{ F3 } & \multicolumn{2}{|c|}{$\mathrm{C} 3$} & \multicolumn{2}{|c|}{ Total } \\
\hline & Masculino & Feminino & Masculino & Feminino & Masculino & Feminino & Masculino & Feminino \\
\hline & n (\%) & n (\%) & n (\%) & n (\%) & n (\%) & n (\%) & n (\%) & n (\%) \\
\hline Epilepsia & $3(7,5)$ & - & $1(3,8)$ & $1(3,8)$ & $3(7,7)$ & - & 8 & $(7,6)$ \\
\hline Trans.mentais & $2(5,0)$ & - & $1(3,8)$ & $1(3,8)$ & $1(2,6)$ & $1(2,6)$ & 6 & $(5,7)$ \\
\hline Total & $29(72,5)$ & $11(27,5)$ & $8(30,8)$ & $18(69,2)$ & $23(59,0)$ & $16(41,0)$ & 105 & $(100)$ \\
\hline \multicolumn{9}{|l|}{ Comorbidades } \\
\hline T.mental dep.tabaco & 17 & 6 & 2 & 12 & 15 & 13 & 65 & $(33,5)$ \\
\hline Obesidade & 6 & 7 & 3 & 7 & 8 & 14 & 45 & $(23,2)$ \\
\hline Hipertensão arterial & 3 & 4 & 2 & 2 & 5 & 5 & 21 & $(10,8)$ \\
\hline Diabetes mellitus & 4 & 1 & - & 1 & 5 & 7 & 18 & $(9,3)$ \\
\hline Epilepsia & 2 & - & - & 5 & 4 & 3 & 14 & $(7,2)$ \\
\hline Retardo mental & 3 & - & 4 & 2 & 1 & 2 & 12 & $(6,2)$ \\
\hline Trans.mentais (outros) & 4 & 1 & & 1 & 1 & 1 & 8 & $(4,1)$ \\
\hline Demais doenças* & 2 & - & - & - & 1 & 2 & 5 & $(2,6)$ \\
\hline $\mathrm{DPOC}^{\dagger}$ & 1 & 1 & - & - & 1 & - & 3 & $(1,6)$ \\
\hline D.Parkinson & - & - & - & - & 2 & - & 2 & $(1,0)$ \\
\hline Hipotireoidismo & - & 1 & - & - & - & - & 1 & $(0,5)$ \\
\hline Total & 42 & 21 & 11 & 30 & 43 & 47 & 194 & $(100)$ \\
\hline
\end{tabular}

*Doença degenerativa, hemiplegia espástica, insuficiência renal crônica

†Doença Pulmonar Obstrutiva Crônica

Foram utilizados 381.564 comprimidos de psicotrópicos com custo de $\mathrm{R} \$ 135.713,01$. Os neurolépticos foram os medicamentos mais consumidos pelos pacientes durante o tratamento, $205.344(53,8 \%)$ comprimidos anualmente, totalizando $\mathrm{R} \$ 128.525,84$. Os medicamentos de uso contínuo não específico para transtornos mentais somaram 68.952 comprimidos perfazendo $\mathrm{R} \$ 5.051,04$ (Tabela 3). Desses, foram mais representativos os antihistamínicos (21.240comp; 30,8\%), antiglicemiantes $(20.520 ; 29,7 \%)$ e anti-hipertensivos $(18.000 ; 26,1 \%)$.

Tabela 3 - Descrição do consumo e custo de medicamentos, segundo unidades de internação (R\$). Lins, SP, Brasil, 2010

\begin{tabular}{|c|c|c|c|c|c|}
\hline \multirow{2}{*}{ Medicamentos } & F1 & \multirow{2}{*}{$\frac{\mathrm{F} 3}{\mathrm{n}}$} & \multirow{2}{*}{$\frac{\mathrm{C} 3}{\mathrm{n}}$} & \multirow{2}{*}{$\begin{array}{c}\text { Total } \\
\text { N (\%) }\end{array}$} & \multirow{2}{*}{$\begin{array}{c}\text { Custo } \\
R \$\end{array}$} \\
\hline & $\mathbf{n}$ & & & & \\
\hline \multicolumn{6}{|l|}{ Psicotrópicos } \\
\hline \multicolumn{6}{|l|}{ Neuroléptico } \\
\hline Típico & 38.880 & 50.928 & 38.676 & $128.484(33,7)$ & $15.324,60$ \\
\hline Atípico & 27.540 & 27.000 & 22.320 & $76.860(20,1)$ & $113.201,24$ \\
\hline Anticonvulsivante & 37.080 & 35.280 & 37.800 & $110.160(28,9)$ & $4.280,40$ \\
\hline Antiparkinsoniano & 9.360 & 6.840 & 13.680 & $29.880(7,8)$ & $1.598,58$ \\
\hline Ansiolítico & 4.680 & 9.720 & 4.860 & $19.260(5)$ & 300,43 \\
\hline Antidepressivo & 4.320 & 1.800 & 2.880 & $9.000(2,4)$ & 293,76 \\
\hline Estabilizador humor & 2.880 & 720 & 4.320 & $7.920(2,1)$ & 714,00 \\
\hline Subtotal & 124.740 & 132.288 & 124.536 & $381.564(100)$ & $135.713,01$ \\
\hline \multicolumn{6}{|l|}{ Outros } \\
\hline Anti-histamínico & 5.760 & 6.120 & 9.360 & $21.240(30,8)$ & 344,08 \\
\hline Antiglicemiante & 3.600 & 2.880 & 14.040 & $20.520(29,7)$ & 619,87 \\
\hline Anti-hipertensivo & 6.840 & 3.240 & 7.920 & $18.000(26,1)$ & 339,23 \\
\hline Laxante & 2.880 & 1.825 & 720 & $5.424(7,9)$ & $3.661,20$ \\
\hline Inib.sec.gástrica & 1.800 & 528 & 1.440 & $3.768(5,5)$ & 86,66 \\
\hline Subtotal & 20.880 & 14.593 & 33.480 & $68.952(100)$ & $5.051,04$ \\
\hline Total & 145.620 & 146.881 & 158.016 & 450.516 & $140.764,05$ \\
\hline
\end{tabular}

Em relação aos indicadores de recursos humanos em enfermagem, a relação enfermagem/leito no período investigado variou de 0,52 a 1,15 , a relação enfermeiro/ leito de 0,05 a 0,11 e a auxiliar de enfermagem/leito de 0,47 a 1,04 (Tabela 4). 
Tabela 4 - Indicadores de recursos humanos de enfermagem no período de 2006 a 2010. Lins, SP. Brasil, 2010

\begin{tabular}{lccccc}
\hline \multicolumn{1}{c}{ Indicadores } & $\mathbf{2 0 0 6}$ & $\mathbf{2 0 0 7}$ & $\mathbf{2 0 0 8}$ & $\mathbf{2 0 0 9}$ & $\mathbf{2 0 1 0}$ \\
\hline $\begin{array}{l}\text { Relação enfermagem/leito } \\
\text { F1 }\end{array}$ & 0,64 & 0,68 & 0,66 & 0,71 & 0,71 \\
F3 & 1,15 & 1,05 & 0,97 & 0,88 & 0,88 \\
C3 & 0,61 & 0,67 & 0,52 & 0,65 & 0,65 \\
Relação enfermeiro*/leito & & & & & \\
F1 & 0,06 & 0,06 & 0,06 & 0,06 & 0,06 \\
F3 & 0,11 & 0,11 & 0,10 & 0,10 & 0,10 \\
C3 & 0,06 & 0,07 & 0,05 & 0,07 & 0,07 \\
Relação auxiliar/leito & & & & & \\
F1 & 0,58 & 0,62 & 0,60 & 0,65 & 0,65 \\
F3 & 1,04 & 0,94 & 0,87 & 0,78 & 0,78 \\
C3 & 0,55 & 0,60 & 0,47 & 0,58 & 0,58 \\
\hline
\end{tabular}

*Enfermeiros ficam lotados em mais de uma unidade

\section{Discussão}

Nos achados deste estudo houve predomínio do sexo masculino $(57,1 \%)$, corroborando outras pesquisas de $53,4 \%{ }^{(12)}$ e $65,5 \%{ }^{(10)}$. É importante destacar que os homens normalmente apresentam alguns distúrbios mentais precocemente, necessitando de internação ${ }^{(11)}$. Evidenciouse que a maioria dos pacientes $(58,1 \%)$ estava na faixa etária entre 41 e 60 anos, diferente de estudos realizados no Rio de Janeiro que encontraram idade inferior a 40 anos $(52,6 \%)^{(11)}$ e entre 30 e 49 anos $(56 \%)^{(12)}$. Chama a atenção o número de pacientes idosos, na faixa etária dos 60 aos 80 anos $(28 ; 26,7 \%)$ semelhante ao valor de $31,3 \%$ encontrado no censo psicossocial, realizado pela Secretaria de Saúde junto a 58 instituições de longa permanência, no Estado de São Paulo ${ }^{(2)}$.

Mostrou-se significativa a quantidade de analfabetos entre os moradores da instituição investigada $(57,1 \%)$, situação também encontrada em outras pesquisas de $50 \%{ }^{(12)}$ e $70 \%{ }^{(2)}$. A baixa escolaridade intensifica a condição de exclusão social ${ }^{(14)}$ e pode ocasionar impacto na adesão ao tratamento por falta de compreensão das orientações realizadas pelos profissionais da saúde ${ }^{(6)}$. Para corrigir essa condição, a instituição oferece oportunidades para que os moradores frequentem o Programa de Alfabetização de Jovens e Adultos (Proaja), o Centro de Educação de Jovens e Adultos (Ceja) ou a Classe Hospitalar. Atualmente, $21 \%$ dos moradores estão matriculados em um desses programas.

Outra característica observada foi de a maioria ser solteira $(89,5 \%)$ e sem fonte de renda $(67,7 \%)$. A dificuldade de interação social pode ter contribuído para essa ocorrência, pois, somado ao fato de apresentar o isolamento como sintoma da doença, há a discriminação e rejeição ao portador de transtorno mental ${ }^{(9)}$.

A esquizofrenia foi o diagnóstico de maior ocorrência (47,7\%), valor semelhante ao encontrado em outros estudos - $43,3 \%^{(10)}$ e $53,6 \%{ }^{(12)}$. Dentre as comorbidades, a elevada porcentagem de tabagismo (62\%) está próxima aos resultados encontrados em Porto Alegre de 38,9 a 54,3\% ${ }^{(15)}$ e em São Paulo de 50 a $84 \%{ }^{(16)}$. A obesidade foi a segunda comorbidade mais presente. Relata-se que a incidência de obesidade, dentre os portadores de esquizofrenia tratados com psicofármacos, é de 40 a $75 \%{ }^{(17)}$.

As medicações antipsicóticas e ansiolíticas foram mais consumidas na unidade F3, por alocar os pacientes com maior número de intercorrências psiquiátricas. Isso evidencia que o tratamento aos portadores de transtornos mentais baseia-se, ainda, no modelo farmacológico ${ }^{(8)}$. Os antipsicóticos estão associados ao aumento importante de peso e alterações metabólicas e, consequentemente, ao aumento do risco de morte por problemas cardiovasculares $^{(18)}$.

O alto custo de um paciente psiquiátrico é evidenciado ao se verificar o consumo das medicações de uso contínuo, tanto psicotrópicas $(\mathrm{R} \$ 135.713,01)$ como as utilizadas para tratamento de problemas clínicos $(\mathrm{R} \$ 5.051,04)$. Porém, é relevante lembrar que, neste estudo foram consideradas somente as medicações de uso contínuo e que esse custo poderia ser alterado ao se incluir todas as medicações consumidas pelo paciente. Investigação mostra que a internação psiquiátrica tem custo mais alto dentre todas as especialidades financiadas pelo Sistema Único de Saúde $(\mathrm{SUS})^{(19)}$

O perfil de pacientes encontrado nesta pesquisa e o longo tempo de internação justificam a existência, ainda, de instituições psiquiátricas de longa permanência, apesar da proposta de desinstitucionalização da Reforma Psiquiátrica. No Brasil, em 2008, os hospitais psiquiátricos totalizavam 32.735 leitos pelo SUS, ou seja, uma proporção de 0,172 leitos por 1.000 habitantes $^{(2)}$. O Estado de São Paulo contava com 58 hospitais psiquiátricos, totalizando 13.190 leitos, sendo 432 leitos em hospitais gerais, com proporção de 0,34 leitos/1.000hab, inferior aos 0,45/1.000 habitantes preconizado pelo Ministério da Saúde; inferior, também, ao encontrado em países como Itália $(4,63)$, Reino Unido $(5,8)$ e Noruega (12), na relação leitos/10.000 habitantes ${ }^{(20)}$.

De acordo com a Organização Mundial da Saúde $(\mathrm{OMS})^{(20)}$, não se consegue a transição de um serviço essencialmente hospitalar para comunitário somente encerrando as instituições psiquiátricas sem proporcionar estruturas alternativas apropriadas. Nos Estados Unidos, a reforma psiquiátrica fez com que aumentasse a quantidade de moradores de rua, por não ocorrer a articulação entre os hospitais psiquiátricos e os serviços comunitários ${ }^{(21)}$. $\mathrm{O}$ conhecimento da população institucionalizada possibilita o planejamento e efetivação de sua reinserção social evitando a falta de assistência.

No que se refere aos indicadores de recursos humanos, a relação entre número de profissionais de enfermagem/leito nas unidades de internação em estudo mostraram variação de 0,52 a 1,15 e a relação enfermeiro/ leito variação de $0,05-0,11$. Os dados apresentados pelo Compromisso com a Qualidade Hospitalar $(\mathrm{CQH})^{(13)}$, referentes ao quarto trimestre de 2009 dos hospitais psiquiátricos que participaram do programa, explicitam a mediana de 0,12 enfermeiros/leito (variação 0,08 a $0,37)$. Esses valores evidenciam que os diversos hospitais psiquiátricos têm diferentes realidades quanto ao pessoal de enfermagem e, na instituição campo de estudo, esse valor é inferior à referenciada. 
Ao enfermeiro compete estabelecer o quadro quantiqualitativo de profissionais necessário para atender as exigências dos pacientes em relação à assistência à saúde ${ }^{(2)}$. Em enfermagem psiquiátrica há poucas discussões de quantos pacientes a equipe de enfermagem consegue assistir terapeuticamente ${ }^{(23)}$, ao contrário de outras especialidades, em que as discussões avançaram muito. As pessoas com transtornos mentais, apesar de não apresentarem, em sua maioria, problemas clínicos que necessitem de assistência de enfermagem constante, requerem observação devido à instabilidade de humor, com ocorrências de auto e heteroagressividade, tentativas de fuga e suicídio ${ }^{(23)}$.

Tornam-se necessários, portanto, estudos adicionais que conduzam a equipe ao quantiqualitativo adequado para não haver comprometimento da qualidade da assistência de enfermagem e da segurança do paciente. Os resultados encontrados nessa instituição podem diferir de outros estudos, sendo necessário dar prosseguimento a novas pesquisas para que a assistência aos portadores de transtornos mentais seja realizada de forma adequada.

\section{Conclusão}

Almeja-se que os resultados dessa investigação possam contribuir para a elaboração de políticas de saúde mental e instrumentalizar o gestor na tomada de decisão e estabelecimento de estratégias de ação.

\section{Referências}

1. Ministério da Saúde (BR). Reforma psiquiátrica e política de saúde mental no Brasil. Documento apresentado à Conferência Regional de Reforma dos Serviços de Saúde Mental: 15 anos depois de Caracas. OPAS. Brasília, novembro 2005.

2. Secretaria da Saúde de São Paulo. Desafios para a desinstitucionalização dos moradores em hospitais psiquiátricos do estado de São Paulo. São Paulo: FUNDAP: Secretaria da Saúde; 2008. 170 p.

3. Joint Commission on Accreditation of Healthcare Organizations (JCAHO). Accreditation manual for hospitals. Nurs Care. 1992 79-85.

4. Bittar OJNV. Indicadores de qualidade e quantidade em saúde. Rev Admin Saúde. 2004; 6(2):15-8.

5. Travassos C, Noronha JC, Martins M. Mortalidade hospitalar como indicador de qualidade: uma revisão. Ciênc Saúde Coletiva. 1999;4(2):367-81.

6. Castro AS de. Caracterização sócio demográfica e clínica das reinternações psiquiátricas no Hospital Santa Tereza de Ribeirão Preto, no período de 2006 a 2007. [dissertação de mestrado]. Ribeirão Preto: Escola de Enfermagem de Ribeirão Preto da Universidade de São Paulo; 2009. 90 p. 7. Fleck MPA, Wagner L, Wagner M, Dias M. Long-stay patients in a psychiatric hospital in Southern Brazil. Rev Saúde Pública. 2007;41(1):124-30.

8. Carvalho MDA, Silva HO, Rodrigues LV. Perfil epidemiológico dos usuários da rede de saúde mental do município de Iguatu, CE. SMAD, Rev. Eletrônica Saúde
Mental Álcool Drog. (Ed. Port.) [Internet]. 2010 [acesso 5 ago 2011]; 6(2):337-49. Disponível em: www2.eerp.usp. br/resmad/artigos/SMAD v6 n2 a7. Pdf.

9. Alves AMO. Perfil sócio demográfico e clínico do portador de doença mental crônica egresso do HospitalDia de Psiquiatria da Santa Casa de Campo Grande-MS. [Dissertação] Campo Grande (MS): Universidade Católica Dom Bosco; 2003.

10. Barros NHS. Características biossociodemográficas e diagnósticos de pacientes internados em hospital psiquiátrico de Campo Grande-MS. [dissertação de mestrado]. Campo Grande (MS): Universidade Católica Dom Bosco. Programa de Mestrado em Psicologia; 2007. 11. Silva JPL, Coutinho ESF, Amarante PD. Perfil demográfico e socioeconômico da população de internos dos hospitais psiquiátricos da cidade do Rio de Janeiro. Cad Saúde Pública. 1999;15:505-11.

12. Gomes MPC, Couto MCV, Pepe VLE, Almeida LM, Delgado PGG, Coutinho ESF. Censo dos pacientes internados em uma instituição asilar no estado do Rio de janeiro: dados preliminares. Cad Saúde Pública. 2002;18(6):1803-7.

13. Programa de Qualidade Hospitalar (CQH). Compromisso com a qualidade hospitalar: apresentação dos indicadores dos hospitais participantes referente ao $4^{\text {o }}$ trimestre de 2009 [Internet]. [acesso 15 out 2011]. Disponível em: http://www.cqh.org.br/?q=node/322 site. pdf

14. Ministério da Educação (BR). Classe hospitalar e atendimento pedagógico domiciliar : estratégias e orientações. Brasília: Secretaria de Educação Especial MEC, SEESP; 2002. 35 p.

15. De Boni R, Pechansky F. Prevalência de tabagismo em uma unidade de internação psiquiátrica de Porto Alegre. Rev Psiquiatria Rio Grande do Sul. 2004;3(25):475-8.

16. Malbergier A, Oliveira HP Júnior. Dependência de tabaco e comorbidade psiquiátrica. Rev Psiquiatr Clin. 2005;32:276-82.

17. Wirshing D. Schizophrenia and obesity: impact of antipsychotic medications. J Clin Psychiatry. 2004;65(Suppl 18):13-26.

18. Colton CW, Manderscheid RW. Congruencies in increased mortality rates, years of potential life lost and causes of death among public mental health clients in eight states. Prev Chronic Dis. [Internet] 2006. Apr [acesso 12 ago 2011]. Disponível em: http://www.cdc.gov/pcd/issues 19. Mello R. A questão da interdisciplinaridade no dia a dia da enfermeira que atua em Centros de Atenção Diária de Saúde Mental. Rev Bras Enferm. 1998;51(1):19-34.

20. Organização Mundial de Saúde. The world health report 2001 mental health: new understanding, new hope. Lisboa (PT); abril 2002.

21. Lovisi VR. Internação psiquiátrica na região de Ribeirão Preto no período de 1989 a 1993. [dissertação de mestrado]. Ribeirão Preto: Escola de Enfermagem de Ribeirão Preto da Universidade de São Paulo; 1998. 96 p. 22. Conselho Federal de Enfermagem (BR). Resolução n.293/2004. Fixa e estabelece parâmetros para o dimensionamento do quadro de profissionais de 
Enfermagem nas unidades assistenciais das instituições de saúde e assemelhados. [acesso 18 ago 2009]. Disponível em: http: //www.corensp.org.br/resolucoes/resolucao293. htm.

23. Martins PASF. Sistema de classificação de pacientes na especialidade enfermagem psiquiátrica [dissertação de mestrado]. São Paulo (SP): Escola de Enfermagem da Universidade de São Paulo; 2001. 